\title{
Expression of Integrin-linked Kinase in Gastric Cancer and Its Relationship with Clinicopathological Characteristics
}

\author{
Jianzhang Hou ${ }^{1,2}$, Yong $\mathrm{Li}^{1,}$ * \\ ${ }^{1}$ Third Department of Surgery, Fourth Hospital of Hebei Medical University, Shijiazhuang, China \\ ${ }^{2}$ Department of Hepatobiliary and Pancreatic (Minimally Invasive) Surgery, Cangzhou People's Hospital, Cangzhou, China
}

Email address:

li-yong-hbth@126.com (Yong Li)

${ }^{*}$ Corresponding author

To cite this article:

Jianzhang Hou, Yong Li. Expression of Integrin-linked Kinase in Gastric Cancer and Its Relationship with Clinicopathological Characteristics. American Journal of Biomedical and Life Sciences. Vol. 8, No. 4, 2020, pp. 119-125. doi: 10.11648/j.ajbls.20200804.18

Received: July 16, 2020; Accepted: August 14, 2020; Published: August 25, 2020

\begin{abstract}
Objective: To explore the value of integrin-linked kinase (ILK) in the diagnosis of gastric cancer, its mechanism of action in the pathogenesis and its influence on tumor metastasis. Methods: Collect 100 cases of gastric cancer diagnosed pathologically, detect the positive expression of ILK, and analyze its biological characteristics. Results: The positive rate of ILK in gastric cancer tissue (57.0\%) is significantly higher than that in the control group (12\%), and the positive rate of ILK gradually increased with the progress of superficial gastritis, chronic atrophic gastritis with atypical hyperplasia and gastric cancer. The differences between the four groups are statistically significant. There is a significant difference in the positive rate of ILK among well-differentiated, moderately-differentiated, poorly-differentiated and undifferentiated adenocarcinomas $(\mathrm{P}<0.001)$. The positive rate $(25 \%)$ of ILK in the uninvaded serosal layer $(\mathrm{T} 1+\mathrm{T} 2)$ is significantly lower than the positive rate $(75.0 \%)$ in the invaded serosal layer (T3+T4). There is a significant difference in the positive rate of ILK in stage I, II, III and IV (P<0.001). In the localized type (I+II), the positive rate of ILK $(24.39 \%)$ is significantly lower than that of the invasive type (III + IV) $(79.66 \%)$. The positive rate of ILK in patients without lymph node metastasis (30.23\%) is significantly lower than that of patients with lymph node metastasis (77.19\%). Conclusion: The positive rate of ILK in gastric cancer tissue is significantly higher than that in adjacent tissues. The higher the positive rate, the worse the prognosis. ILK is an independent risk factor affecting the prognosis of patients.
\end{abstract}

Keywords: Gastric Cancer, Degree of Differentiation, Borrmann Classification, TNM Staging, Lymph Node Metastasis, Integrin-Linked Kinase

\section{Introduction}

Gastric cancer (GC) is one of the most common gastrointestinal malignant tumors in the clinic. Its morbidity and mortality are second only to lung cancer. New cases of gastric cancer in our country account for more than $17 \%$ of all malignant tumors, and mortality accounts for more than $20 \%$ [1], ranking third among all malignant tumors [2]. The incidence of gastric cancer has the characteristics of hidden incidence, low consultation rate, often missed diagnosis, easy metastasis and high recurrence. Most patients are in the advanced stage at the time of treatment. The proportion of radical surgical resection is not high, and the incidence of postoperative recurrence and metastasis is high. The median survival time is only 3 to 24 months, and the 5 -year survival rate is less than $30 \%(20 \%$ to $50 \%)$. The treatment effect is poor, and it has become one of the malignant tumors that seriously threaten human health [3]. So far, the mechanism of occurrence and development of gastric cancer has still not been very clear. Researches have shown that integrin-linked kinase (ILK) is an important effector in the signal transduction pathway of integrin and growth factor receptors. It has multiple biological functions and participates in cell proliferation, differentiation, growth, the regulation of adhesion, migration, infiltration and tumor angiogenesis. Therefore, ILK is closely related to the occurrence and 
development of tumors, especially invasiveness [4]. ILK is rarely expressed in normal human tissues, but it is highly expressed in neurogenic tumors, lung cancer, melanoma, ovarian cancer, prostate cancer, gastric cancer, and colon cancer, and its expression level gradually increases with the increase of tumor pathological grade. It is significantly related to the malignancy and metastasis of gastric cancer, prostate cancer, ovarian cancer and small cell lung cancer [5]. The research on ILK in gastric cancer has been reported, but there is no consistent conclusion on the results, and the changes in gastric cancer and its pathogenesis are unclear. In this research, 100 primary gastric cancer tissues diagnosed by histopathology are used as the object. The immunohistochemical method (SP method) is used for detecting the expression of ILK in specimens of gastric cancer, normal gastric mucosa and adjacent tissues, analyzing the relationship and correlation with the clinicopathological characteristics, invasion, and metastasis of gastric cancer, and exploring the diagnostic value of gastric cancer and its role in the pathogenesis of gastric cancer and its impact on tumor metastasis, which provides theoretical basis for early diagnosis, prognosis and targeted therapy of gastric cancer.

\section{Information and Methods}

\subsection{Research Object}

A total of 100 patients with primary gastric cancer diagnosed for the first time from the Department of Gastrointestinal Surgery, Cangzhou People's Hospital, Department of General Surgery, Cangzhou Central Hospital, and Department of External Medicine, Cangzhou Integrated Traditional Chinese and Western Medicine Hospital Histopathologically from January 2016 to October 2017, all patients with gastric cancer do not undergo chemotherapy or radiotherapy before surgery. 100 cases of gastric cancer tissues removed by surgery. 40 cases of normal gastric mucosa ( $5.0 \mathrm{~cm}$ away from the edge of the tumor) tissues, 40 cases of superficial gastritis with gastroscope biopsy, and 20 cases of chronic atrophic gastritis with dysplasia are placed in $10 \%$ of neutral formalin buffer that fixed for $24 \mathrm{~h}$, and embedded in paraffin. Three specimens of each are serially sectioned, with a thickness of $4 \mu \mathrm{m}$. One of them is subjected to routine tissue HE staining for pathological analysis, and the other 2 are subjected to ILK immunohistochemical staining. Clinical data is collected in detail, which includes the patient's gender, age, tumor size, tumor location, Lauren classification, degree of differentiation, depth of invasion, TNM staging, Borrmann classification, lymph node metastasis, etc. There are 69 males and 31 females. The age ranges from 31 to 78 years, including 21 cases $<60$ years old and 79 cases $\geq 60$ years old, with an average age of (65.62 \pm 10.56$)$ years. It is grouped according to the "Diagnosis and Treatment of Gastric Cancer (Ministry of Health-2011 Edition)" [6], Borrmann classification [7] and the 2016 International Anti-Cancer Alliance/United Cancer Council (UICC/AJCC) TNM staging standard [8]. 100 primary gastric cancer patients in the research object, including (1) gender: 69 males and 31 females; (2) age: 32 patients $<60$ years old, 68 patients $\geq 60$ years old; (3) tumor size: 41 cases with diameter $<5.0 \mathrm{~cm}, 59$ cases with $\geq 5.0 \mathrm{~cm}$; (4) tumor site: 49 cases of gastric antrum, 27 cases of stomach body, 24 cases of stomach bottom; (5) Lauren classification: diffuse type 51 Cases, 35 cases of intestinal type, 14 cases of mixed type; (6) degree of differentiation: 41 cases of poorly differentiated and undifferentiated adenocarcinoma, 32 cases of moderately differentiated adenocarcinoma, 27 cases of highly differentiated adenocarcinoma; (7) depth of invasion: uninvaded 36 cases with serous layer $(\mathrm{T} 1+\mathrm{T} 2), 64$ cases with invasion of serous layer and out of serous layer (T3+T4); (8) TNM staging: 11 cases in stage I, 26 cases in stage II, 44 in stage III Cases, 19 cases of stage IV; (9) Borrmann classification: 41 cases of localized type (I+II), 59 cases of invasive type (III+IV); (10) lymph node metastasis: 43 cases without lymph node metastasis, with lymph node metastasis 57 cases.

\subsection{Research Methods}

\subsubsection{Experimental Instruments and Reagents}

Main instruments: JY-TSF dehydrator and JY-TK slice bleaching and drying instrument (domestic), Histocore Arcadia H/C embedding machine, RM2235 slicer, BOND-MAX automatic immunohistochemistry instrument (Germany Leica) and OLYMPUS Bx51 optical microscope (Japan). Main reagents: ILK (rabbit anti-human monoclonal antibody) [EP1593Y] (ab52480) Abcam (UK), purchased from Fuzhou Maixin Biotechnology Development Co., Ltd., rabbit anti-human S100P polyclonal antibody [EPR6143] (ab133554) (ready to use Type) is Abcam (UK), goat anti-rabbit secondary antibody (ready-to-use) rapid immunohistochemistry Max Vision TM detection kit, purchased from Fuzhou Maixin Biotechnology Development Co., Ltd.

\subsubsection{Immunohistochemical Staining}

Using immunohistochemistry (SP) method for gastric cancer tissues, normal gastric mucosa tissues $>5.0 \mathrm{~cm}$ from the tumor margin and superficial gastritis obtained by gastroscopy biopsy and chronic atrophic gastritis with dysplastic tissue Perform ILK immunohistochemical staining. Rabbit anti-human ILK and SP immunohistochemical stains and DAB chromogens are purchased from Fuzhou Maixin Biotechnology Development Company, using known positive tissue samples as positive controls, and using $\mathrm{pH}$ 7.4 PBS instead of primary antibodies as negative controls. The staining procedure is strictly in accordance with the instructions of the kit. The specific steps are as follows: According to the routine procedures of pathological technology, slide processing, section preparation, immunohistochemical staining and neutral gum mounting are performed, and then the immunohistochemical sections are observed and photographed with a photographic microscope.

\subsubsection{Judgment of Results}

Using PBS instead of primary antibody as a negative 
control, and known positive sections as a positive control. Using an optical microscope, the staining of the cells is observed under a high magnification microscope, and the cytoplasm and/or nucleus showed light yellow or brown particles as positive. ILK positive is mainly expressed in the cytoplasm, and a small amount is expressed on the cell membrane. According to the presence or absence of positive particles and color, the intensity of cell staining is divided into 4 levels. The scoring criteria are: 0 for unstained, 1 for light yellow, 2 for yellow or dark yellow, and 3 for tan. Randomly select 5 high-power fields and count the average number of positive cells according to the staining range. The percentage of positive cells is divided into 3 levels: 0 points: no positive cells; 2 points: the positive cell rate is $26 \%-50 \%, 3$ Score: Positive cell rate $>51 \%$. The judgment criterion of the staining result is: the product of the staining intensity and the percentage score of positive cells, that is, the final score of each sample: 0 to 2 points are negative (-), and 3 to 6 points are positive $(+)$. When the overall result is judged, $\leq 2$ points are negative and $>2$ points are positive. According to Wang et al [9] scoring criteria, cells with no positive staining or positive staining $<5 \%$ are negative, and those with positive cell staining $>5 \%$ are positive.

\subsection{Statistical Methods}

The obtained clinical data and ILK immunohistochemical staining results are sorted out. All data are analyzed by SPSS21.0 statistical software. The relationship between the results of ILK immunohistochemical staining and clinical parameters is analyzed by $\chi 2$ test. The counted data is expressed as a percentage. The correlation analysis is taken by Spearman rank correlation test. If the test results demonstrate $\mathrm{P}<0.05$, which indicate that the difference has statistically significant.

\section{Results}

\subsection{Expression of ILK in Gastric Cancer Tissues}

The positive rate of ILK in gastric cancer tissues $(57.0 \%, 57 / 100)$ is significantly higher than that in the control group $(12 \%, 12 / 100)$. The differences have statistically significant $(\chi 2=45.74, \mathrm{P}<0.001)$, and with the development of superficial gastritis, chronic atrophic gastritis with dysplasia and gastric cancer. The positive rate of ILK gradually increases, and the differences among the four groups have statistically significant. The rate is significantly higher than that of chronic atrophic gastritis with dysplasia, superficial gastritis and normal gastric mucosa tissue. The difference has statistically significant $(\mathrm{P}<0.05)$, however, there is no significant difference in the positive rate of ILK among chronic atrophic gastritis with dysplasia, superficial gastritis and normal gastric mucosa $(\mathrm{P}>0.05)$. See Figures 1-4 $(\times 200)$, Table 1 .

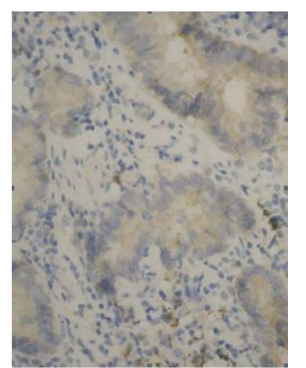

Figure 1. Positive expression of ILK in normal gastric mucosa.

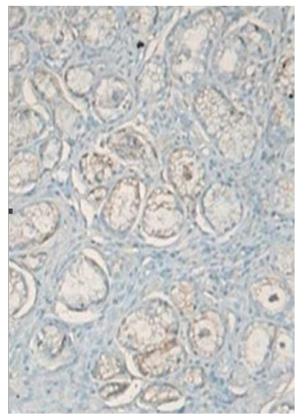

Figure 2. Positive expression of ILK in Superficial gastritis tissues.

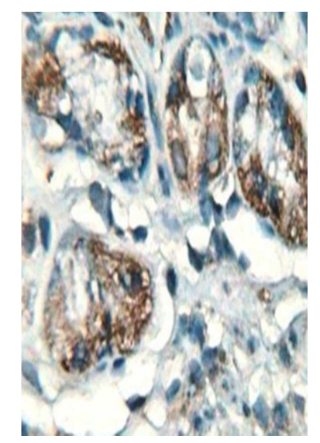

Figure 3. Positive expression of ILK in Chronic atrophic gastritis with atypical hyperplasia tissues.

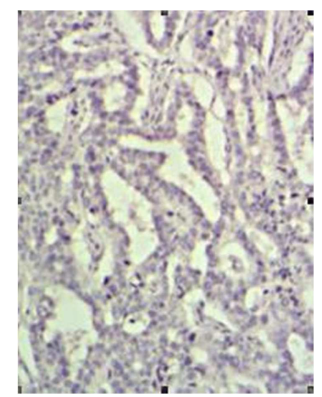

Figure 4. Positive expression of ILK in gastric carcinoma tissues.

Table 1. Comparison of positive rates of ILK in gastric carcinoma tissues and control.

\begin{tabular}{llllc}
\hline Groups (n) & ILK Positive (\%) & ILK Negative (\%) & $\chi^{2}$ & $\boldsymbol{P}$ \\
\hline I group (100) & $57(57.0)$ & $43(43.0)$ & & \\
II group (20) & $4(20.0)$ & $16(80.0)$ & 45.735 & $<0.001$ \\
III group (40) & $5(12.5)$ & $35(87.5)$ & & \\
IV group (40) & $3(7.5)$ & $37(92.5)$ & & \\
\hline
\end{tabular}

Note: Group I (gastric cancer group), Group II (chronic atrophic gastritis with dysplasia), Group III (superficial gastritis), Group IV (normal gastric mucosal tissue). 
The positive rate of ILK between gastric cancer and the control group has statistically significant $(\chi 2=45.735, p<0.001)$, but the positive rate of ILK between the control group has not statistically significant $(\mathrm{P}>0.05)$. There are 6 groups comparing each other, $\mathrm{P}=0.05 / 6=0.008$. Compared with group I and II, $\mathrm{x}^{2}=9.129, \mathrm{P}=0.003$. Compared with group I and III, $\mathrm{x}^{2}=7.652$, $\mathrm{P}=0.006$. Compared with group $\mathrm{I}$ and $\mathrm{IV}, \mathrm{x}^{2}=28.586, \mathrm{P}=0.000$. Compared with group II and III, $\mathrm{x}^{2}=0.588, \mathrm{P}=0.443$. Compared with group II and IV, $\mathrm{x}^{2}=2.022, \mathrm{P}=0.155$. Compared with group III and IV, $\mathrm{x}^{2}=0.556, \mathrm{P}=0.456$.

\subsection{The Relationship Between ILK Expression and Clinicopathological Characteristics of Patients with Gastric Cancer}

There is no significant difference in the positive rate of ILK in gastric cancer tissues with the gender, age, tumor size and location of patients and Lauren classification $(\chi 2$ values are $0.021,0.011,0.317,0.197$ and 3.054, $P$ values are 0.885, 0.917, $0.574,0.906$ and 0.217 respectively), but the positive rate of ILK in diffuse gastric cancer tissues $(64.71 \%, 33 / 51)$ is higher than intestinal type $(45.71 \%, 16 / 35)$. In the degree of differentiation of gastric cancer, the positive rates of ILK in well differentiated, moderately differentiated, poorly differentiated and undifferentiated adenocarcinomas are $25.93 \%$ $(7 / 27), 46.88 \%(15 / 32), 85.37 \%(35 / 41)$. There is a significant difference among the groups $(\chi 2=25.435, \mathrm{P}<0.001)$; At the depth of infiltration, the positive rate of $\operatorname{ILK}(25 \%, 9 / 36)$ that does not invade the serous layer $(\mathrm{T} 1+\mathrm{T} 2)$ is significantly lower than that of the invasion positive rate $(75.0 \%, 48 / 64)$ of serous layer and invading serous layer $(\mathrm{T} 3+\mathrm{T} 4)$. There is a significant difference between the two groups $(\chi 2=23.501$, $\mathrm{P}<0.001$ ); In TNM stage, the positive rates of ILK, I, II, III and IV are $18.18 \%(2 / 11), 34.62 \%(9 / 26), 68.18 \%(30 / 44)$ and $84.21 \%(16 / 19)$. Among 4 groups, there is a significant difference $(\chi 2=20.062, \mathrm{P}<0.001)$; In Borrmann classification, the positive rate of localized (I+II) ILK $(24.39 \%, 10 / 41)$ is significantly lower than that of infiltrated type (III+IV) positive rate $(79.66 \%, 47 / 59)$. There is a significant difference between the two groups $(\chi 2=31.150, \mathrm{P}<0.001)$; In lymph node metastasis, the positive rate of ILK without lymph node metastasis $(30.23 \%, 13 / 43)$ has significant. It is lower than the positive rate of lymph node metastasis $(77.19 \%, 44 / 57)$, and there is a significant difference between the two groups $(\chi 2=22.053, \mathrm{P}<0.001)$. See Table 2.

Table 2. Relationship between the expression of ILK and clinicopathological features in gastric carcinoma tissues.

\begin{tabular}{|c|c|c|c|c|c|}
\hline Clinicopathological features & example & Negative & Positive (\%) & $\chi^{2}$ & $P$ \\
\hline \multicolumn{6}{|l|}{ Gender } \\
\hline Male & 69 & & $39(56.52)$ & \multirow[t]{2}{*}{0.021} & \multirow[t]{2}{*}{0.885} \\
\hline Female & 31 & 13 & $18(58.06)$ & & \\
\hline \multicolumn{6}{|l|}{ Age } \\
\hline$<60$ & 32 & 14 & $18(56.25)$ & \multirow[t]{2}{*}{0.011} & \multirow[t]{2}{*}{0.917} \\
\hline$\geq 60$ & 68 & 29 & $39(57.35)$ & & \\
\hline \multicolumn{6}{|l|}{ Tumor size $(\mathrm{cm})$} \\
\hline$<5$ & 41 & 19 & $22(53.66)$ & \multirow[t]{2}{*}{0.317} & \multirow[t]{2}{*}{0.574} \\
\hline$\geq 5$ & 59 & 24 & $35(59.32)$ & & \\
\hline \multicolumn{6}{|l|}{ Tumor location } \\
\hline Antrum & 49 & 20 & $29(59.18)$ & \multirow{3}{*}{0.197} & \multirow{3}{*}{0.906} \\
\hline Stomach body & 27 & 12 & $15(55.56)$ & & \\
\hline Fundus & 24 & 11 & $13(54.17)$ & & \\
\hline \multicolumn{6}{|l|}{ Differentiation } \\
\hline low & 41 & 6 & $35(85.37)$ & \multirow{3}{*}{25.435} & \multirow{3}{*}{$<0.001$} \\
\hline middle & 32 & 17 & $15(46.88)$ & & \\
\hline high & 27 & 20 & $7(25.93)$ & & \\
\hline \multicolumn{6}{|l|}{ Depth of infiltration } \\
\hline T1/T2-lessthan serosa & 36 & 27 & $9(25.00)$ & \multirow[t]{3}{*}{23.501} & \multirow[t]{2}{*}{$<0.001$} \\
\hline T3/T4-Slurry and beyond & 64 & 16 & $48(75.00)$ & & \\
\hline \multicolumn{5}{|l|}{ TNM staging } & \\
\hline I & 11 & 9 & $2(18.18)$ & \multirow{4}{*}{20.062} & \multirow{4}{*}{$<0.001$} \\
\hline II & 26 & 17 & $9(34.62)$ & & \\
\hline III & 44 & 14 & $30(68.18)$ & & \\
\hline IV & 19 & 3 & $16(84.21)$ & & \\
\hline \multicolumn{6}{|l|}{ Lauren typing } \\
\hline Diffuse & 51 & 18 & $33(64.71)$ & \multirow{4}{*}{3.054} & \multirow{3}{*}{0.217} \\
\hline Gut type & 35 & 19 & $16(45.71)$ & & \\
\hline Hybrid & 14 & 6 & $8(57.14)$ & & \\
\hline \multicolumn{5}{|l|}{ Borrmann typing } & \\
\hline $\mathrm{I}+\mathrm{II}$ & 41 & 31 & $10(24.39)$ & \multirow[t]{3}{*}{31.150} & \multirow[t]{3}{*}{$<0.001$} \\
\hline III+IV & 59 & 12 & $47(79.66)$ & & \\
\hline \multicolumn{4}{|l|}{ Lymph node metastasis } & & \\
\hline No & 43 & 30 & $13(30.23)$ & 22.053 & $<0.001$ \\
\hline Have & 57 & 13 & 44 (77.19) & & \\
\hline
\end{tabular}




\section{Discussion}

Gastric cancer is one of the most common gastrointestinal malignant tumors in China, and its morbidity and mortality are relatively high. In 2015, the incidence and mortality of gastric cancer in our country are as high as $679.1 / 100,000$ and $498.0 / 100,000$, respectively, and the age of onset is becoming more and more younger. The prognosis is poor, and the 5 -year survival rate of patients is less than $30 \%$, which seriously affects human health and the quality of life of patients [10-13]. Molecular genetic studies have found that gastric cancer, as a highly heterogeneous malignant tumor, whose occurrence and development is a complex process involving multiple stages and multiple genes. It explores, finds and screens the molecules for early diagnosis, occurrence, metastasis and prognosis of gastric cancer. Markers will have an important role in promoting the targeted therapy of gastric cancer genes.

ILK is a serine/threonine (Ser/Thr) protein kinase with three structural units first discovered by Hannigan and others in the double-hybrid screening of yeast in 1996. Its relative molecular mass is 59000 [18], and the full length of gene is $8.8 \mathrm{~kb}$, which is located on chromosome $11 \mathrm{p} 5.5-11 \mathrm{p} 15.4$, and including 12 introns and 13 exons, of which the first 12 exons are involved in translation. ILK is an integrin-binding protein. The molecule contains 3 domains: N-terminal has 4 ankyrin repeats (ANK), which can be combined with focal interesting new cysteine histidine rich protein (PIHCH) and ILK binding protein (ILK associated protein, ILKAP). PIHCH has a localization and function regulating effect on ILK, and ANK can mediate the interaction between ILK and ILK-related phosphatase (ILKAP); the middle is the phosphatidylinositol binding domain ( $\mathrm{PH}$ domain), connected to phosphoinositide lipid and bounding to phosphatidylinositol 3,4,5-triphosphate (PIP3); C-terminal is protein kinase domain, ILK phosphorylates with 3-hydroxyphosphatidylinositol through its own phosphorylation. The combination of the product of alcohol kinase (PIP3) and the binding of the PH domain to PIP3 are activated to play different biological roles. Activated ILK can phosphorylate protein kinase B (PKB) Ser473 and glycogen synthase kinase (GSK3) to be activated, coordinate the signal transduction of extracellular matrix (ECM) and growth factors, and regulate cell growth, differentiation and migration, etc. [13]. Researches have shown that ILK, as an important effector in integrin and growth factor receptor signaling pathways, has multiple biological functions, whose related proteins GSK3 and PKB are important regulators of cell cycle and apoptosis. It is also involved in the regulation of cell proliferation, differentiation, growth, adhesion, migration, infiltration and tumor angiogenesis. Therefore, ILK is closely related to tumor formation, tumor cell adhesion, invasion and metastasis. As a multi-domain protein, ILK not only plays a role as a molecular scaffold in the adhesion between cells and ECM, but also participates in cell apoptosis, migration and invasion through various signaling pathways. Overexpression or continuous activation of ILK can lead to cell-independent adherence-dependent survival, tumorigenic transformation, add to tumorigenicity, and enhance tumor invasiveness.
Conversely, inhibiting ILK expression can reduce PKB/Akt activity, induce cell cycle arrest and apoptosis, thereby inhibiting tumor growth [14]. Therefore, the expression of ILK is closely related to the development and treatment of tumors, confirming the dependence of tumor cells on ILK enzyme activity and conversion function during tumor progression. ILK antisense oligonucleotide therapy may be to inhibit tumor progression Effective preparation [15]. ILK can dephosphorylate PIP3 to PIP2 through PH domain, protein tyrosine phosphatase and tensin homolog (PTEN), antagonize PI3K signal transduction, inactivate or delete PTEN, leading to many tumors. The occurrence of ILK has become an important target for the treatment of many tumors. Edwards LA et al. [16] found that the combined application of ILK antisense oligonucleotides and anti-tumor drugs had a clear synergistic effect, which opened up a new way for gene therapy targeting ILK and show a broader application prospect.

Ito et al. [17] reported that ILK was highly expressed in gastric cancer tissues, and its expression level was positively correlated with the depth of tumor invasion and lymph node metastasis, while ILK was lowly expressed in normal gastric mucosa tissues. Zhang Fengmei et al. [18] found that the positive rate of ILK in gastric cancer tissues $(55.0 \%, 44 / 80)$ was significantly higher than that in the control group $(6.7 \%$, $2 / 30, \mathrm{P}<0.05)$, and increased with the depth of tumor invasion. In patients with lymph node and omentum metastasis, the positive rate of ILK was significantly higher than those without metastasis, which suggested that ILK may be closely related to the development of gastric cancer and play an important role in invasion and metastasis. Dong Liang [19] reported that the positive rate of ILK in gastric cancer tissues $(72.5 \%, 29 / 40)$ was significantly higher than chronic atrophic gastritis with dysplasia $(35.0 \%, 7 / 20)$ and superficial gastritis group $(10.0 \%, 2 / 20)$. The difference between the two pairs had statistically significant, and ILK was highly expressed in gastric cancer tissues with lymph node metastasis, Borrmann type III/IV and TNM stage III/IV $(\mathrm{P}<0.05)$, which was related to tumor progression. However, there was no significant correlation with gender, age, degree of differentiation, and depth of infiltration $(\mathrm{P}>0.05)$. There was no statistically significant difference in the positive rate of ILK between benign gastric diseases. According to the Spearman correlation analysis, it was found that ILK and Hsp 90 in gastric cancer tissue were moderately significantly positively correlated $(0.5<\mathrm{r} \leq 0.8 ; \mathrm{P}=0.000)$, both of which were potential targets for gastric cancer chemotherapy. The high expression of ILK was significantly related to Borrmann classification, lymph node metastasis and clinical stage, which suggested that it was related to the progress of gastric cancer, but Hsp 90 had no obvious correlation with the pathological parameters of gastric cancer, which suggested that ILK was more specific than Hsp 90 in the role of gastric cancer cells. This research found that the positive rate of ILK (57.0\%) in gastric cancer tissue was significantly higher than that in the control group $(\mathrm{P}<0.05)$, and gradually increased with the changes of NGM, SG, CAG with dysplasia and GC, GC group 
with CAG. There was no significant difference between the dysplasia groups, but there was significant difference with other groups $(\mathrm{P}<0.05)$; the positive rate of ILK in gastric cancer tissues was closely related to the degree of tumor differentiation, depth of invasion, TNM stage, Borrmann classification, and lymph node metastasis $(\mathrm{P}<0.05)$. In patients with poor differentiation, deep infiltration, late stage and metastasis, the positive rate of ILK was higher, which was related to the clinical severity, histological malignancy and prognosis of gastric cancer, and was related to the gender of the patient, age, tumor size, tumor location has nothing to do. It suggested that the overexpression of ILK was involved in the occurrence and development of gastric cancer. Overexpression of ILK in tumor tissue may activate certain tumor-related factors and their signal transduction pathways, thereby participating in the occurrence and development of gastric cancer, and playing an important role in their invasion and metastasis.

There are few researches on ILK in Lauren classification of gastric cancer. Lauren classification is one of the simple and effective classification methods for gastric cancer. It is the most commonly used histological classification standard for GC [20], and the consistency between different researchers is relatively high. Lauren P divided GC into intestinal gastric cancer (IGC) and diffuse gastric cancer (DGC) based on the organizational structure and biological behavior of GC in 1965, which is the Lauren classification of gastric cancer. IGC generally has obvious glandular cavity formation, often produced on the basis of intestinal metaplasia, and mostly grows locally. Cancer cells are mostly columnar or cuboid, which mainly compose of high and medium differentiated glands, and are of different sizes. Like structure, some cancer cells are like goblet cells of intestinal metaplasia, and sometimes poorly differentiated gland components can be seen at the front of infiltration; the adjacent mucosa is often accompanied by extensive atrophic gastritis and intestinal metaplasia. DGC generally has no glandular cavity formation, and is scattered in the stomach wall due to poor adhesion of tumor cells, lacking intercellular connection, and most of them growing diffusely, mainly poorly differentiated adenocarcinoma or signet ring cell carcinoma, and cancer cells are often poorly differentiated small round, which can also be a single cell cord, incomplete glandular or mesh structure, occasionally a small amount of interstitial mucus, accompanied by obvious interstitial fibrous connective tissue hyperplasia; no or only a small piece of atrophic gastritis and intestinal metaplasia. About $10 \%$ to $15 \%$ of GC has both intestinal and diffuse characteristics, that is, when the ratio of the two is equivalent, it is called a mixed type. The Lauren classification of GC has been used until now. According to the classification of WHO (2010) [21], IGC includes papillary adenocarcinoma and tubular adenocarcinoma; DGC includes signet ring cell carcinoma and poorly differentiated adenocarcinoma [22-23]. DGC and IGC have difference in etiology, pathogenesis, epidemiology, treatment methods, and prognosis. Cao Wenjun [24] found that the clinical characteristics and prognosis of GC patients with different Lauren classifications were different, and believed that the classification played a certain role in the treatment and prevention of gastric cancer. Yuan Qianqian et al [25] reported that the differences between DGC and IGC in gender, age, tumor diameter, $\mathrm{T}$ stage, $\mathrm{N}$ stage, and TNM stage had statistically significant $(\mathrm{P}<0.05)$. DGC was more likely to occur in young women than IGC. The larger the tumor volume, the more aggressive it was, the easier it was to develop lymph node metastasis. The later the stage, the worse the prognosis than IGC. Univariate survival analysis showed that age, tumor diameter, location, $\mathrm{T}$ stage, $\mathrm{N}$ stage, TNM stage, and Lauren classification were related factors that affected the prognosis of GC. Multivariate survival analysis showed that age, tumor location, N stage, TNM stage, and Lauren classification were independent factors that affected the prognosis of GC. Therefore, it is believed that Lauren classification can not only reflect the biological behavior of $\mathrm{GC}$, but also reflect the etiology, pathogenesis and epidemiological characteristics of GC. This research has found that the positive rate of ILK in DGC tissue is slightly higher than that of IGC, and its change trend is consistent with the above research results, but in general, there is no significant difference in the expression of ILK in Lauren classification of gastric cancer $(\mathrm{P}>0.05)$. Therefore, the application of ILK in Lauren classification of gastric cancer needs to be prospectively studied in a large sample.

The results show that the positive rate of ILK in gastric cancer tissues is significantly higher than that in adjacent tissues. The lower the degree of differentiation, the deeper the infiltration, the later the TNM stage and Borrmann classification, and those with lymph node metastasis, the higher the positive rate of ILK. It indicates that the high expression of ILK may be involved in the development of gastric cancer and is related to the invasion and metastasis of gastric cancer, which may provide a basis for the early diagnosis, targeted therapy and metastasis of gastric cancer. The positive expression of ILK is obviously related to the prognosis of gastric cancer. The higher the positive rate, the worse the prognosis. It is an independent risk factor that affects the prognosis of patients and can be used as an indicator to judge the prognosis of patients. The positive rate of ILK in gastric cancer tissue has nothing to do with the patient's gender, age, tumor size, tumor location and Lauren classification. In short, exploring the pathogenesis of gastric cancer from the molecular biological level, looking for effective early diagnostic indicators, and regulatory genes that affect tumor cell proliferation, apoptosis, invasion and metastasis, and exploring possible signaling mechanisms at the molecular level, looking for new gene therapy and molecular targets that affect prognosis have become a hot spot in gastric cancer research. In-depth research on the occurrence, development and treatment of gastric cancer is of great significance for improving the quality of life and survival rate of gastric cancer patients.

\section{Conclusion}

The positive rate of ILK in gastric cancer tissue is significantly higher than that in adjacent tissue. The lower the 
degree of differentiation, the deeper the infiltration, the later the TNM stage and Borrmann classification, and those with lymph node metastasis, the higher the positive rate of ILK. The positive expression of ILK is obviously related to the prognosis of gastric cancer. The higher the positive rate, the worse the prognosis. It is an independent risk factor that affects the prognosis of patients. The positive rate of ILK in gastric cancer tissue has nothing to do with the patient's gender, age, tumor size, tumor location and Lauren classification.

\section{References}

[1] Ra Fiei E, Mohammadian-Hafshejani A, Towhidi F, et al. Lack of Any relation-ship of Stomach Cancer Incidence and Mortality with development in Asia [J]. Asian Pacific J cancer Prevention, 2016, 17 (8): 3777-3783.

[2] Lam JK, Chow MY, Zhang Y, et al. siRNA Versus miRNA as Therapeutics for Gene Silencing [J]. Mol Ther Nucleic Acids, 2015, 4 (9): 252.

[3] Chen WQ, Zheng RS, Zhang SW, et al. Report of Cancer Incidence and Mortality in China, 2012, Chin Canc, 2016, 25 (1): $1-8$.

[4] Li N, Li YF, Wu XF. Advances in biological function of integrin-linked kinase [J]. Life Chem, 2016, 36 (3): 385-389.

[5] Qiang ZR, Yang GD, Wu J, et al. The role of integrin-linked kinases in tumorigenesis and progression $[\mathrm{J}]$. Chin Clin Onco, 2006, 11 (11): 873-875.

[6] Department of Medical Administration, Ministry of Health of the People's Republic of China. Guidelines for the Diagnosis and Treatment of Gastric Cancer (2011 edition) Chin J Frontiers Med Scie (Electronic Version) 2012, 4 (5): 62-71.

[7] Song J, Dong SL, Duan JP, et al. Expression of SDF-1/CXCR4 in gastric carcinoma of different Borrmann type and its clinical significances [J]. Chin J Clin (Electronic Edition) 2015, 9 (20): 3691-3695.

[8] M. B. Amin et al. American Joint Committee on Cáncer A JCC Cáncer Staging M anual, Eighth Edition [J], 2017 203-220.

[9] Wang G Platt-Higgins A, Carroll $\mathrm{J}$, et al. Induction of metastasis by $\mathrm{S} 100 \mathrm{P}$ in a rat mammary model and its association with poor survival of breast cancer patients $[\mathrm{J}]$. Cancer Res, 2006, 66 (2): 1199-1207.

[10] Laks S, Meyers MO, Kim HJ. Surveillance for Gastric Cancer [J]. Surg Clin North Am. 2017, 97 (2): 317-331.
[11] Chen W, Zheng R, Baade PD, et al. Cancer statistics in China, 2015 [J]. CA Cancer J Clin. 2016, 66 (2): 115-132.

[12] Strand MS, Lockhart AC, Fields RC. Genetics of Gastric Cancer [J]. Surg Clin North Am. 2017, 97 (2): 345-370.

[13] Ilson DH. Current Progress in the Adjuvant Treatment of Gastric Cancer [J]. Surg Oncol Clin N Am. 2017, 26 (2): 225-239.

[14] Gao J, Zhu J, Li HY, et al. Small interfering RNA targeting integrin-linked kinase inhibited the growth and induced apoptosis in human bladder cancer cells [J]. Int J Biochem Cell Biol. 201143 (9): 1294-1304.

[15] Xiong T, Qu Y, Mu DZ. Integrin-Linked KinaseandIntegrin [J]. J Appl Clin Pedi, 2009, 24 (23): 1848-1851.

[16] Edwards LA, Shabbits JA, Bally M, et a1. Integrin-linked kinase (ILK) in combination molecular targeting [J]. Cancer Treat Res, 2004, 119 (1): 59-75.

[17] Ito R, Oue N, Zhu X, et al. Expression of integrin-linked kinase is closely correlated with invasion and metastasis of gastric carcinoma [J]. Virchows Arch, 2003, 442 (2): 118-123.

[18] Zhang FM, Li SS, Li XQ, et a1. Expression and significance of ILK and MMP-9 in gastric cancer invasion and metastasis [J]. Chin J Gastr Hepa, 2015, 24 (1): 95-98.

[19] Dong L. Expression and significance of Hsp90 and its client protein ILK in gastric cancer and precancerous Iesion [J]. Chin J Integr Trad West Med Dig, 2016, 24 (4): 271-275.

[20] Hu B, El Hajj N, Sittler S, et al. Gastric cancer: Classification, histology and application of molecular pathology [J]. Gastrointest Oncol, 2012, 3 (3): 251-261.

[21] Li ZS, Li Q. WHO Classification of Digestive System Tumors in 2010 edition [J]. Chin J Pathol, 2011, 40 (5): 351-354.

[22] Xie J, Fang J, Jin ML, et al. Advances in pathological typing of gastric cancer [J]. Chin J Prac Inter Medi, 2014, 34 (6): 626-630.

[23] Hu JK, Chen XZ. Treatment and Prognosis Based on Types and Stages of Gastric Cancer [J]. Chin J Bases Clin General Surg.

[24] Cao WJ, Li M, LI HJ. Clinical characteristics and prognosis of intestinal- type and diffuse-type gastric cancer [J]. Chin Gene Prac, 2017, 20 (13): 1587-1591.

[25] Yuan QQ, Ye HZ, Xu LP, et al. Clinicopathological characteristics and prognosis of gastric carcinoma by Lauren classification [J]. Acta Acade Medicinae Wannan, 2016, 35 (6): 544-547. 